\title{
Thermal Sunyaev-Zel'dovich anisotropy due to primordial black holes
}

\author{
Katsuya T. Abe, ${ }^{*}$ Hiroyuki Tashiro, and Toshiyuki Tanaka \\ Division of Particle and Astrophysical Science, Graduate School of Science, \\ Nagoya University, Chikusa, Nagoya 464-8602, Japan
}

\begin{abstract}
We investigate the thermal Sunyaev-Zel'dovich (SZ) effect caused by primordial black holes (PBHs) on the cosmic microwave background (CMB) temperature fluctuations. The gas accreting on a PBH heats up by the release of the gravitational energy. As a result, the heated gas in the vicinity of the PBH emits UV and x-ray photons. These photons can ionize and heat the intergalactic medium (IGM) around the PBH. Assuming the simple model of these emitting photons, we compute the profiles of the IGM ionization fraction and temperature around a PBH by using the numerical calculation of the radiative transfer. Using these profiles, we evaluate the Compton $y$-parameter created by the IGM gas around a PBH. Finally, we estimate the CMB temperature angular power spectrum due to the PBH SZ effect in our model. We show that the SZ temperature anisotropy due to the PBHs has the flat angular power spectrum on small scale, $l \leq 2000$ and could dominate the primordial temperature spectrum on smaller scales than the Silk scale. This flat spectrum extends to the scale of the ionized region by the $\mathrm{PBH}$ emission. Therefore, in future SZ anisotropy measurements, the detection or nondetection of the flat spectrum gives useful information about the existence of PBHs.
\end{abstract}

\section{INTRODUCTION}

Many astronomical observations including the motion of stars in a galaxy, the large-scale structure distribution, and the cosmic microwave background (CMB) temperature anisotropy indicate the existence of dark matter (DM) in the Universe. However, its nature is still poorly understood. Currently, since the presence of DM can be probed only through its gravitational interaction, observational information about its nature is very limited. However, there are many works to study the nature of DM and many theoretical models have been suggested so far. Generally, the DM candidates are classified into two types; the nonbaryonic particle model and astrophysical compact object model. A lot of models for the nonbaryonic particle type are predicted in the physics beyond the standard model such as weakly interacting massive particles [1], axions [2-5] and axionlike particles [6]. On the other hand, in the latter type, a primordial black hole $(\mathrm{PBH})$ is the most potential candidate [7].

$\mathrm{PBHs}$ could have formed from high-density peaks in the very early Universe $[8,9]$. In the inflation paradigm, the density fluctuations are generated from the quantum fluctuations. When an overdense region which exceeds a critical density threshold enters the horizon scale, the gravitational collapse of this region happens to form a $\mathrm{PBH}$. The resultant mass of the formed $\mathrm{PBH}$ corresponds the horizon mass at the horizon-crossing epoch of the overdense region. As a result, the $\mathrm{PBH}$ mass range can span very widely. Therefore, the $\mathrm{PBH}$ abundance has been studied for a long time not only for DM but also as the relic of the primordial density fluctuations on small scales. Besides, the recent detection of gravitational wave $(\mathrm{GW})$ events drew attention to PBHs as sources

* abe.katsuya@e.mbox.nagoya-u.ac.jp of GWs. The analysis of the observed GW data reveals that the detected GW events were produced by binary black hole mergers with masses larger than $20 M_{\odot}[10$ 12]. It would be difficult to produce a black hole with such large mass from stellar evolution in the standard solar metallicity environment [13]. On the other hand, the broad mass range of $\mathrm{PBH}$ can cover the observed black hole masses. Therefore, the PBH abundance is also studied as a responsible source for the detected GW events $[14-16]$.

The existence of $\mathrm{PBHs}$ affects various cosmological phenomena, depending on the PBH mass. For small mass PBHs, the abundance can be constrained by the effects of their evaporation. As first pointed out by Hawking [17], a black hole emits many kinds of particles with the thermal spectrum. As a result, $\mathrm{PBH}$ s with a mass smaller than $10^{15} \mathrm{~g}$ have evaporated by the present epoch. The abundance constraint on evaporated PBHs is obtained by investigating the effect of their evaporation on big bang nucleosynthesis [18], the CMB spectrum distortion [19], the recombination and reionization processes [20, 21], and the diffuse gamma-ray background [22-24].

For nonevaporated PBHs, the robust constraint is provided by gravitational lensing observations [25-27]. The black hole merger rate evaluated from the recent detection of GW events also constrains the $\mathrm{PBH}$ abundance $[15,16]$. Recently several works focused on the gas accreting on massive PBHs. Because of the release of the gravitational energy during accretion, the gas becomes hot and emits x-ray and UV photons [28-30]. Resultantly the surrounding gas around a PBH is heated and ionized. Studying the cosmological effects of such heating and reionization provides the constraint on stellar mass PBHs with the recent CMB measurement [28-31]. There are also some works suggesting that future $21-\mathrm{cm}$ observations can probe these $\mathrm{PBH}$ heating and ionizing processes and give the strong constraint on $\mathrm{PBHs}$ in this mass range $[32,33]$. 
In this paper, we study the thermal SunyaevZel'dovich (SZ) effect due to PBHs. The thermal SZ effect is the distortion of the CMB energy spectrum through the inverse Compton scattering by high-energy electrons in hot plasma and this effect is parametrized by the Compton $y$-parameter [34, 35]. As mentioned above, $\mathrm{PBH}$ can heat and ionize the surrounding gas. The resultant ionized gas contributes not only to the global optical depth of the Thomson scattering as discussed in Refs. [28-30], but also to creating the nonzero Compton $y$-parameter. The sky-averaged (global) Compton $y$ paremter is already evaluated in Ref. [29]. They have obtained the global Compton $y$-parameter induced by $\mathrm{PBH}$ from evaluating the energy deposit of the $\mathrm{PBH}$ luminosity into a plasma. According to their estimation, the global Compton $y$-parameter could be roughly $y \sim 10^{-7} f_{\mathrm{PBH}}$, where $f_{\mathrm{PBH}}$ describes the $\mathrm{PBH}$ abundance fraction to the total DM one. Since this estimated value is below the sensitivity of the Far-Infrared Absolute Spectrophotometer [36], they concluded that it is difficult to obtain a constraint on the $\mathrm{PBH}$ abundance from the global Compton $y$-parameter with the current observation status.

Here, we focus on the spatial fluctuations of the Compton $y$-parameter induced by $\mathrm{PBH}$ s and the resultant CMB temperature anisotropy due to them. The angular power spectrum of the CMB temperature anisotropy could provide additional information through its amplitude and shape depending on scales which we cannot access from the global value. Our main aim is to study the dependence of the SZ angular power spectrum on the $\mathrm{PBH}$ properties such as the abundance, mass and so on.

To evaluate the spatial fluctuations of the Compton $y$-parameter, it is required to obtain the profiles of the ionization faction and the intergalactic medium (IGM) gas temperature around a $\mathrm{PBH}$. Introducing the emission efficiency parameter of the PBH luminosity, we evaluate these profiles through the one-dimensional radiative transfer calculation. Then, assuming that the PBH distribution follows the dark matter density fluctuations, we calculate the CMB temperature anisotropy due to the SZ effect of PBHs. We investigate the dependence of the $\mathrm{PBH}$ fraction, the $\mathrm{PBH}$ mass and the emission efficiency parameter on the generated CMB anisotropy. From the comparison with the small-scale measurement of the CMB temperature anisotropy by South Pole Telescope (SPT) [37], we also discuss the suggestion of the $\mathrm{SZ}$ effect due to $\mathrm{PBH}$ s on the constraint on the $\mathrm{PBH}$ abundance with the emission efficiency parameter.

The rest of this paper is organized as follows. In Sec. II, we compute the gas temperature and ionization fraction around a $\mathrm{PBH}$. Accordingly, we calculate the profile of the thermal SZ effect. In Sec. III, introducing the $\mathrm{PBH}$ fraction to dark matter, we evaluate the $\mathrm{CMB}$ temperature angular power spectrum due to the thermal SZ effect around PBHs. We also discuss the $\mathrm{PBH}$ abundance constraint from comparing our results with the SPT data. Finally, we summa- rize in Sec. IV. Throughout our paper, we take the flat $\Lambda$ CDM model with the Planck best fit parameters [38]: $\left(\Omega_{\mathrm{m}}, \Omega_{\mathrm{b}}, h, n_{\mathrm{s}}, \sigma_{8}\right)=(0.32,0.049,0.67,0.97,0.81)$.

\section{THERMAL SUNYAEV-ZEL'DOVICH EFFECT DUE TO A PBH}

In this section, we evaluate the gas temperature and ionization fraction of the IGM around a PBH, assuming the photon energy spectrum emitted from the hot gas in the vicinity of a $\mathrm{PBH}$. Accordingly, we calculate the profile of the thermal SZ effect.

\section{A. The luminosity from a $\mathbf{P B H}$}

Since a PBH creates a gravitational potential, the surrounding gas accretes on the $\mathrm{PBH}$. During accretion, as it goes closer to a $\mathrm{PBH}$, the gas becomes hot enough to emit x-ray and UV photons [28-30]. However, since the gas temperature highly depends on the astrophysical processes and environmental condition, there is a theoretical uncertainty in this luminosity. References [28, 29] evaluated the luminosity of a $\mathrm{PBH}$ considering spherical accretion on the $\mathrm{PBH}$ and showed that the luminosity is much lower than the Eddington luminosity. On the other hand, Ref. [30] pointed out that a PBH could have an accretion disk and that its luminosity is much higher than in the case of the spherical accretion. Moreover, when a massive PBH has an accretion disk, its luminosity might become nearly sub-Eddington luminosity similar to the active galactic nuclei as considered in Ref. [39].

Therefore, this emission efficiency can depend on the redshift, the $\mathrm{PBH}$ mass and other physical conditions around the $\mathrm{PBH}$. Here, for simplicity, we introduce one free parameter for the emission efficiency, $\epsilon$, which represents the total $\mathrm{PBH}$ luminosity, $L_{\mathrm{PBH}}$, in terms of the Eddington luminosity.

$$
L_{\mathrm{PBH}}=\epsilon L_{\mathrm{Edd}},
$$

where $L_{\mathrm{Edd}}$ is the Eddington luminosity for mass $M$

$$
L_{\mathrm{Edd}}=3.2 \times 10^{4} L_{\odot}\left(M / M_{\odot}\right) .
$$

We also assume the power-law type of luminosity spectrum:

$$
L_{\mathrm{PBH}, \nu}=\mathcal{A} \nu^{-1.5}
$$

where $\mathcal{A}$ is determined by

$$
L_{\mathrm{PBH}}=\int_{\nu_{\mathrm{L}}} L_{\mathrm{PBH}, \nu} d \nu,
$$

with the Lyman limit frequency, $\nu_{\mathrm{L}}$. In Eq. (3), we take the frequency spectral index of -1.5 similar to that in the case of galactic black holes [40]. 


\section{B. IGM temperature and ionization profiles around a $\mathrm{PBH}$}

Now we consider the profiles of the ionization fraction and gas temperature around a $\mathrm{PBH}$ with $L_{\mathrm{PBH}}$. For simplicity, we consider only hydrogen as the IGM gas component.

The evolutions of the ionization fraction, $x_{\mathrm{e}}$ and temperature $T_{\text {gas }}$ in the IGM are given by the following equations,

$$
\begin{aligned}
& \frac{d x_{\mathrm{e}}}{d t}=k_{\mathrm{HI}, \gamma}-\alpha_{\mathrm{B}} n_{\mathrm{H}} x_{\mathrm{e}}^{2}, \\
& \frac{d \mathrm{~T}_{\mathrm{gas}}}{d t}=(\gamma-1) \frac{\mu m_{\mathrm{p}}}{k_{\mathrm{B}} \rho}\left(\frac{k_{\mathrm{B}} T_{\mathrm{gas}}}{\mu m_{\mathrm{p}}} \frac{d \rho}{d t}+\Gamma-\Lambda\right),
\end{aligned}
$$

where $n_{\mathrm{H}}, k_{\mathrm{B}}, \gamma, \mu, m_{\mathrm{p}}, \rho$ and $\alpha_{\mathrm{B}}$ are the number density of hydrogen nucleus, the Boltzmann constant, the IGM gas adiabatic index, $\gamma=5 / 3$, the mean molecular weight, the proton mass, the gas mass density, and the case B recombination rate given in Ref. [41] respectively. In Eqs. (5) and (6), $k_{\mathrm{HI}, \gamma}, \Gamma$ and $\Lambda$ are the ionization, heating and cooling rates, respectively. Here, we adopt cooling mechanisms due to recombination cooling, collisional ionization cooling, collisional excitation cooling and Compton cooling [41]. For simplicity, we assume that the IGM gas is homogeneous and we set the IGM gas density to the cosmological mean (background) values at a given time $t$.

We consider only photons emitted from a $\mathrm{PBH}$ as the ionization and heating source. We solve Eqs. (5) and (6) with the spherically symmetric assumption. Since we evaluate the ionization and temperature evolutions for the cosmological timescale, it is useful to introduce the comoving radial distance from a $\mathrm{PBH}$ for representing the spatial position, instead of the physical distance. The ionization and heating rates at the comoving radial distance $r$ can be written, respectively, as

$$
\begin{aligned}
k_{\mathrm{HI}, \gamma}(r) & =\left(1-x_{\mathrm{e}}(r)\right) \int_{\nu_{\mathrm{L}}}^{\infty} \mathcal{F}_{\nu}(r) \frac{\sigma_{\mathrm{HI}, \nu}}{h \nu} d \nu, \\
\Gamma(r) & =n_{\mathrm{HI}}(r) \int_{\nu_{\mathrm{L}}}^{\infty} \mathcal{F}_{\nu}(r) \frac{\left(\nu-\nu_{\mathrm{L}}\right)}{\nu} \sigma_{\mathrm{HI}, \nu} d \nu,
\end{aligned}
$$

where $h, n_{\mathrm{HI}}(r)$ and $\sigma_{\mathrm{HI}, \nu}$ are the Planck constant, the neutral hydrogen number density given in $n_{\mathrm{HI}}(r)=$ $\left(1-x_{\mathrm{e}}(r)\right) n_{\mathrm{H}}$, and the absorbed cross section area of ionization photons, $\sigma_{\mathrm{HI}, \nu}=6.3 \times 10^{-18}\left(\nu / \nu_{\mathrm{L}}\right)^{-3} \mathrm{~cm}^{2}$, respectively. In the above equations, $\mathcal{F}_{\nu}(r)$ represents the photon energy flux for a frequency $\nu$ at a comoving distance $r$ :

$$
\mathcal{F}_{\nu}(r)=\frac{L_{\mathrm{PBH}, \nu}}{4 \pi a^{2}(t) r^{2}} e^{-\tau_{\mathrm{HI}, \nu}(r)} .
$$

Here $a(t)$ is the scale factor normalized as $a\left(t_{0}\right)=1$ at the present epoch, $t_{0}$, and $\tau_{\mathrm{HI}, \nu}(r)$ is the optical depth of $\mathrm{HI}$ gas from the central PBH to the comoving distance $r$,

$$
\tau_{\mathrm{HI}, \nu}(r)=\int_{0}^{r} a(t) n_{\mathrm{HI}}\left(r^{\prime}\right) \sigma_{\mathrm{HI}, \nu} d r^{\prime} .
$$

Solving Eqs. (5) and (6) consistently by a spherically symmetric radiative transfer code, we obtain the evolutions of $x_{\mathrm{e}}$ and $T_{\text {gas }}$ at each comoving distance $r$ and cosmic time $t$.

Figure. 1 shows the radial profile of the ionization fraction as a function of the physical distance, $R=a(t) r$, from a $\mathrm{PBH}$. In the figure, we set $M=10 M_{\odot}$ and $\epsilon=$ 0.0001. The difference of colors represents different redshifts. As the Universe evolves, the ionized region extends outward. This expansion is proportional to $(1+z)^{-2}$. This behavior is consistent with the redshift evolution of the Strömgren radius with the constant photon flux from a $\mathrm{PBH}$. The Strömgren radius $R_{\mathrm{s}}$ (in physical) can be related to the number rate of photons emitted from a $\mathrm{PBH}, N_{\mathrm{PBH}, \gamma}$, and has the redshift dependence as in

$$
R_{\mathrm{s}}=\left(\frac{3 N_{\mathrm{PBH}, \gamma}}{4 \pi n_{\mathrm{e}} n_{\mathrm{p}} \alpha_{\mathrm{B}}}\right)^{1 / 3} \propto(1+z)^{-2},
$$

where $N_{\mathrm{PBH}, \gamma}$ is obtained from

$$
N_{\mathrm{PBH}, \gamma}=\int_{\nu_{\mathrm{L}}}^{\infty} d \nu \frac{L_{\mathrm{PBH}, \nu}}{h \nu} .
$$

Since we assume that the photon emission from a $\mathrm{PBH}$ does not have the redshift evolution, the redshift dependence in Eq. (11) comes from the (physical) number density of electrons and protons, $n_{\mathrm{e}}, n_{\mathrm{p}} \propto(1+z)^{3}$.

Figure 2 provides the redshift evolution of the IGM gas temperature. We plot the radial profile of the temperature as a function of the physical radial distance from a PBH. The color difference shows the difference of the redshifts. Similarly to the ionization fraction in FIG. 1, the lower the redshift becomes, the more the volume of the heated region increases. In the fully ionized central region, the temperature profile is flat and the redshift dependence of the amplitude is very weak. Since the heating mechanism is photoionization, the heating becomes efficient in the neutral region. Therefore, the peak of the gas temperature locates at the spatial position where the ionization fraction becomes about $x_{\mathrm{e}} \simeq 0.1$. Thus, the heated region extends more than the ionized region. In a sufficiently distant region, the gas temperature becomes the background IGM temperature which is represented by the thin dashed lines in Fig. 2.

At the end of this subsection, we comment on the dependence of the ionization fraction and gas temperature on the $\mathrm{PBH}$ luminosity, $L_{\mathrm{PBH}} \propto \epsilon M$. According to Eq. (11) with Eq. (12), the Strömgren radius depends on $(\epsilon M)^{1 / 3}$. As shown in Figs. 1 and 2, the profiles of $x_{\mathrm{e}}$ and $T_{\text {gas }}$ is sensitive to the evolution of the Strömgren radius. Therefore, as $\epsilon$ or $M$ increases, the ionization front expands towards outside roughly proportional to $(\epsilon M)^{1 / 3}$. However, since the temperature is determined by the balance between the heating and cooling, the amplitude of the temperature at the fully ionized region does not have the simple dependence on $\epsilon M$. 


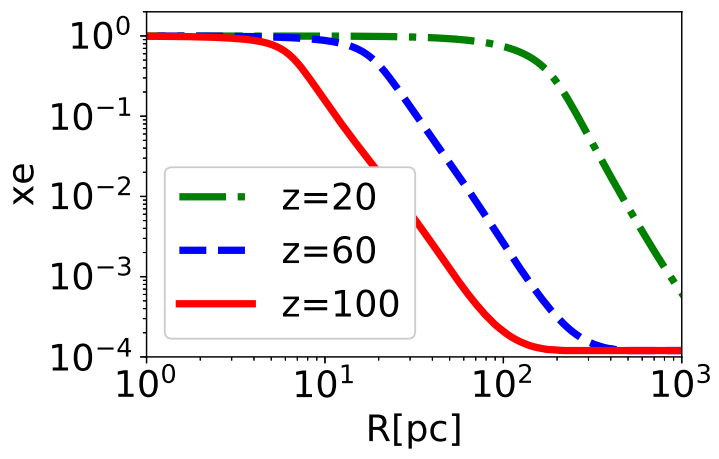

FIG. 1. The radial ionization fraction profile around a $\mathrm{PBH}$ as a function of the physical distance from the $\mathrm{PBH}$. We set $M=10 M_{\odot}$ and $\epsilon=0.0001$ in this figure. The red solid, blue dashed and green dashed-dotted lines represent the profiles at $z=100, z=60$ and $z=20$, respectively.

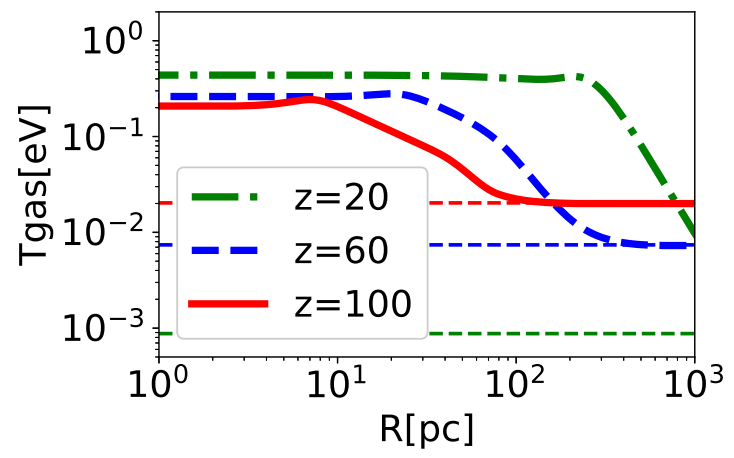

FIG. 2. The radial temperature profile around a $\mathrm{PBH}$ as a function of physical distance from the $\mathrm{PBH}$. We set $M=$ $10 M_{\odot}$ and $\epsilon=0.0001$ in this figure. The meaning of the color is the same as in Fig. 1. The thin dashed lines represent the background IGM gas temperature at each redshift.

\section{Compton $y$-parameter induced by a $\mathbf{P B H}$}

Figures. 1 and 2 show that a $\mathrm{PBH}$ can make a hot gas plasma around itself. Passing through this plasma, CMB photons suffer from the SZ effect. As a result, the observed brightness temperature of the CMB is shifted by $\Delta T_{\nu}$ at a frequency $\nu$ from the background CMB temperature, $T_{\mathrm{CMB}}[34]$. At the comoving distance $b$ from the $\mathrm{PBH}$ on the sky, this observed shift of the brightness temperature can be written with the Compton $y$-parameter,

$$
\frac{\Delta T_{\nu}(b)}{T_{\mathrm{CMB}}}=g(\nu) y(b),
$$

where $g(\nu)$ is the frequency spectral function of the SZ effect,

$$
g(\nu)=\frac{h \nu}{k_{\mathrm{B}} T_{\text {gas }}} \tanh ^{-1}\left(\frac{h \nu}{2 k_{\mathrm{B}} T_{\text {gas }}}\right) .
$$

The Compton $y$-parameter at the comoving distance $b$ on the sky, $y(b)$, can be obtained from the integral form along a line-of-sight with the impact parameter $b$ from the $\mathrm{PBH}$,

$$
y(b)=\int d x \frac{\sigma_{\mathrm{T}} n_{\mathrm{H}} x_{\mathrm{e}}(\ell)}{m_{\mathrm{e}} c} k_{\mathrm{B}} T_{\mathrm{gas}}(\ell),
$$

where $\sigma_{\mathrm{T}}$ is the Thomson cross section, $m_{\mathrm{e}}$ is the electron mass and $c$ is the speed of light. In Eq. (15), $x$ is the comoving distance projected on the line-of-sight direction and $\ell$ is the comoving radial distance from the $\mathrm{PBH}$ satisfying, $\ell^{2}=b^{2}+x^{2}$.

Using Eq. (15) with the profiles of the ionization fraction and temperature shown in Figs. 1 and 2, we can compute the $y$-parameter. We plot the $y$-parameter due to a $\mathrm{PBH}$ with $M=10 M_{\odot}$ and $\epsilon=0.0001$ as a function of the physical distance, $R_{\mathrm{b}}=a(t) b$, for different redshifts in Fig. 3. As expected, the $y$-parameter profile depends on the ionization fraction profile. Therefore, when $R_{\mathrm{b}}$ becomes larger than the Strömgren radius, the $y$-parameter quickly falls down. Following the evolution of the Strömgren radius, as the Universe evolves, the flat region in the $y$-parameter profile extends outward. In Eq. (15), most of the contribution to the integration come from the region inside the Strömgren radius (the fully ionized region).

The amplitude of $y$-parameter decreases as the Universe evolves because the redshift dependence of the electron number density dominates that of the integral distance and gas temperature.

We also comment on the dependence of the $y$ parameter profile on $\epsilon$ and $M$. As mentioned above, the $y$-parameter profile strongly depends on the Strömgren radius. Since the Strömgren radius is proportional to $(\epsilon M)^{1 / 3}$, the parameters $\epsilon$ and $M$ can affect the $y$ parameter profile. As $\epsilon$ or $M$ becomes large, the amplitude of the $y$-parameter increases and the tail of the profile moves toward large $R_{\mathrm{b}}$.

Based on Fig. 3, we can roughly estimate the total $y$-parameter induced by $\mathrm{PBHs}$ on the line-of-sight direction between $z=100$ and $z=20$. Figure 3 shows that one $\mathrm{PBH}$ with $M=10 M_{\odot}$ and $\epsilon=10^{-4}$ provides $y \simeq 10^{-12}$. Since the comoving number density of such $\mathrm{PBH}$ is about $n_{\mathrm{PBH}} \sim 10^{9} f_{\mathrm{PBH}} \mathrm{Mpc}^{-3}$, where $f_{\mathrm{PBH}}$ is the $\mathrm{PBH}$ fraction to the total DM abundance, the resultant typical distance of $\mathrm{PBHs}$ is roughly $10^{-3} \mathrm{Mpc}$. Since the radial distance between $z=100$ and $z=20$ is about $10^{3} \mathrm{Mpc}$, the PBH number on the line-of-sight is $N_{\mathrm{PBH}} \sim 10^{6}$. Therefore, the total $y$-parameter integrated over the line-of-sight is $y \sim 10^{-6}$. Consequently, this $\mathrm{y}$ parameter can generate a SZ signature on the order of $\mu \mathrm{K}$. In the next section, we evaluate the anisotropic signal in more detail. 


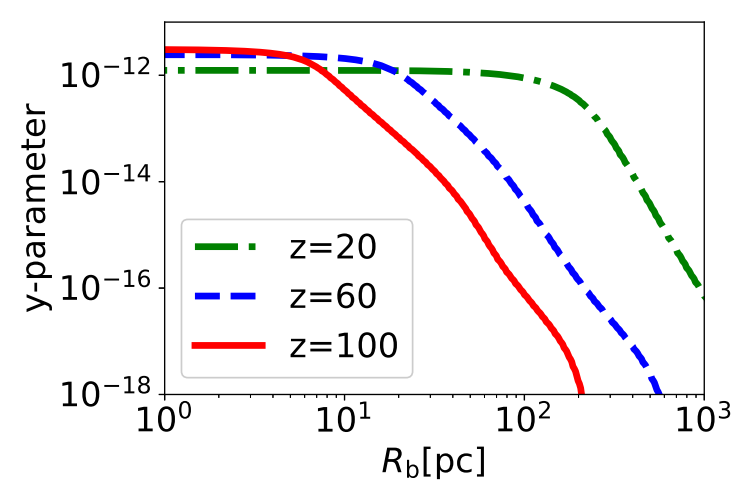

FIG. 3. The Compton $y$-parameter as a function of the physical impact parameter from the $\mathrm{PBH}$. We set $M=10 M_{\odot}$ and $\epsilon=0.0001$ in this figure. The meaning of the color is the same as in Fig. 1.

\section{THERMAL SUNYAEV-ZEL'DOVICH ANISOTROPY DUE TO PBHS}

As shown in the previous section, a hot plasma is generated around a $\mathrm{PBH}$ and can cause the $\mathrm{SZ}$ effect. If $\mathrm{PBH}$ account for a significant fraction of the dark matter abundance, they can produce the observable CMB temperature anisotropy through the SZ effect. In this section, we evaluate the angular power spectrum of the CMB temperature fluctuations by the SZ effect. We also discuss the constraint on the $\mathrm{PBH}$ abundance from the comparison with the small-scale CMB measurement from the SPT.

\section{A. The CMB temperature angular power spectrum by the SZ effect}

To calculate the angular power spectrum of the CMB temperature due to the SZ effect caused by $\mathrm{PBHs}$, we take a similar method to the one for galaxy clusters based on the halo formalism $[42,43]$. Accordingly, the angular power spectrum can be described as the sum of the two contributions:

$$
C_{l}^{T T}=g^{2}(\nu)\left(C_{l}^{y y(1 \mathrm{P})}+C_{l}^{y y(2 \mathrm{P})}\right),
$$

where $C_{l}^{y y(1 \mathrm{P})}$ is the "one-PBH" term describing the Poisson contribution and $C_{l}^{y y(2 \mathrm{P})}$ is the "two-PBH" term arising from clustering of $\mathrm{PBH}$. Assuming that the $\mathrm{PBH}$ mass function is restricted to a single mass $M$, we can write these two terms as

$$
\begin{aligned}
C_{l}^{y y(1 \mathrm{P})} & =\int_{z_{\mathrm{f}}}^{z_{\mathrm{ini}}} d z \frac{d^{2} V}{d z d \Omega} n_{\mathrm{PBH}}\left|y_{l}(z)\right|^{2}, \\
C_{l}^{y y(2 \mathrm{P})} & =\int_{z_{\mathrm{f}}}^{z_{\mathrm{ini}}} d z \frac{d^{2} V}{d z d \Omega} P\left(\frac{l}{d(z)}\right) n_{\mathrm{PBH}}^{2}\left|y_{l}(z)\right|^{2},
\end{aligned}
$$

where $V$ is the comoving volume, $d(z)$ is the comoving distance to the redshift $z, P(k)$ is the matter power spectrum and $n_{\mathrm{PBH}}$ is the comoving number density of $\mathrm{PBHs}$ with mass $M$. Here we assume that PBHs contribute to the DM abundance with the fraction $f_{\mathrm{PBH}}$. Therefore, $n_{\mathrm{PBH}}$ can be written as $n_{\mathrm{PBH}}=f_{\mathrm{PBH}} \Omega_{\mathrm{DM}} \rho_{\text {crit }} / M$ with the present critical density of the Universe, $\rho_{\text {crit }}$. We integrate these equations from the redshift $z_{\text {ini }}$ to $z_{\mathrm{f}}$ for whose values we will add small discussion later.

In Eqs. (17) and (18), taking the small angle approximation, we get $y_{l}$ as the two-dimension Fourier transform of the Compton $y$-parameter for $\mathrm{PBH}$ mass $M$ at the redshift $z$ obtained in Sec. II C:

$$
y_{l}(z)=\int d^{2} \theta y(b) \exp (-i \boldsymbol{\theta} \cdot \boldsymbol{l}),
$$

where $\boldsymbol{l}$ is a vector describing the two-dimensional Fourier mode with $l=|\boldsymbol{l}|$, the comoving distance $b$ on the sky is given in $b=|\boldsymbol{\theta}| d(z)$ and $\boldsymbol{\theta}$ is the angular direction on the sky sphere.

Calculating Eqs. (17) and (18) with the profile of $y(b)$, we can obtain the CMB temperature anisotropy induced by the $\mathrm{SZ}$ effect due to PBHs. In Fig. 4, we plot the obtained angular power spectra of the $\mathrm{CMB}$ temperature anisotropy for the different $\mathrm{PBH}$ parameter sets $\left(M, \epsilon, f_{\mathrm{PBH}}\right)$. Our $\mathrm{PBH}$ parameter sets are summarized in Table I. For comparison, we show the primordial CMB temperature anisotropy as the black dashed-dotted line. We also plot the SPT data with the error bars [37].

The shape of the spectrum is independent on the $\mathrm{PBH}$ parameters. We find out that the contribution from the two-PBH term dominates the one-PBH term. Since the typical scale of the $y$-parameter profile in Fig. 3 is the Strömgren radius, $R_{\mathrm{s}}$, which is much smaller than the CMB observation scales, $y_{l}$ is constant on the CMB observation scales. Therefore, according to Eq. (18), the shape of the angular spectrum is determined by the matter power spectrum. In fact, independently on the $\mathrm{PBH}$ parameters, Fig. 4 shows that the spectrum has a flat shape on larger multipoles than $l \sim 2000$. Because of this flat shape, the SZ temperature anisotropy due to $\mathrm{PBHs}$ can dominate the primordial temperature anisotropy on smaller scales than the Silk scale.

On the other hand, the amplitude depends on the $\mathrm{PBH}$ parameters. Because of the integration range in Eq. (15), $y(b)$ is proportional to $R_{\mathrm{s}}$. Figure 3 tells us that the typical scale of non-negligible $y(b)$ is also $R_{\mathrm{s}}$. Therefore, according to Eq. (19), the angular Fourier component of the $y$-parameter, $y_{l}$, is proportional to $R_{\mathrm{s}}^{3} \propto \epsilon M$. The $\mathrm{PBH}$ number density is $n_{\mathrm{PBH}} \propto f_{\mathrm{PBH}} / M$. As a result, $C_{l}$ has the dependence as $C_{l} \simeq C_{l}^{y y(2 \mathrm{P})} \propto\left|n_{\mathrm{PBH}} y_{l}\right|^{2} \propto$ $\left(\epsilon f_{\mathrm{PBH}}\right)^{2}$ and we found that the angular power spectrum on the flat region $l>2000$ can be approximated in

$$
l^{2} C_{l} \sim 0.5 \times\left(\frac{f_{\mathrm{PBH}}}{10^{-2}}\right)^{2}\left(\frac{\epsilon}{10^{-4}}\right)^{2} .
$$

Figure 4 clearly shows this dependence of $C_{l}$ to us. Because of the degeneracy between $\epsilon$ and $f_{\mathrm{PBH}}$, the angular 


\begin{tabular}{|c|c|c|c|}
\hline & $M\left[M_{\odot}\right]$ & $\epsilon$ & $f_{\mathrm{PBH}}$ \\
\hline \hline (A) & 10 & $1.0 \times 10^{-4}$ & $7.6 \times 10^{-2}$ \\
\hline (B) & 100 & $1.0 \times 10^{-4}$ & $7.6 \times 10^{-2}$ \\
\hline (C) & 1000 & $1.0 \times 10^{-4}$ & $7.6 \times 10^{-2}$ \\
\hline (D) & 10 & $1.0 \times 10^{-2}$ & $7.6 \times 10^{-4}$ \\
\hline (E) & 100 & $1.0 \times 10^{-2}$ & $7.6 \times 10^{-4}$ \\
\hline (F) & 1000 & $1.0 \times 10^{-2}$ & $7.6 \times 10^{-4}$ \\
\hline
\end{tabular}

TABLE I. The PBH parameter sets for the CMB anisotropy due to PBHs in Fig. 4.

spectrum for the case $\mathrm{C}$ is coincident with that for the case D.

We also investigate the redshift contribution to the angular spectrum. The observed temperature anisotropy is a resultant effect which is integrated along the line-ofsight direction. As already mentioned, $y_{l}$ is proportional to $R_{s}^{3}$ which becomes large as the Universe evolves. In particular, we find out that the contribution from the redshift higher than $z=50$ is less than $1 \%$. On the other hand, in the low-redshift side, the Universe is gradually reionized and finally fully ionized until $z \sim 7$ [38]. During the epoch of reionization, the regions ionized by PBHs are caught up in the reionization process by stars and galaxies. As a result, in lower redshifts around the epoch of reionization, the $\mathrm{SZ}$ effect due to $\mathrm{PBHs}$ becomes fainter as the reionization proceeds. Here, for simplicity, we take into account the PBH SZ effect between $z_{\text {ini }}=200$ and $z_{\mathrm{f}}=10$ in the integration of Eqs. (17) and (18).

In Ref. [29], they calculated the typical value of $\epsilon$ in the analytic model with the spherical accretion on a $\mathrm{PBH}$. Their obtained $\epsilon$ is on the order of $10^{-9}$ for the $\mathrm{PBH}$ mass $100 M_{\odot}$ at $z \sim 10$. Substituting this value into Eq. (20), we can see that the signal is much smaller than the current observation sensitivity such as the SPT even for $f_{\mathrm{PBH}}=1$. However, there exists the theoretical uncertainty in $\epsilon$ as mentioned in Sec. II. Depending on the environment around a PBH, $\epsilon$ could become larger than the above one. In that case, we might get the observable value in the present observation status. Therefore, in the next subsection, we discuss the possible constraint on the PBH abundance from the thermal SZ angular power spectrum with the free parameter $\epsilon$.

\section{B. Application to the $\mathrm{PBH}$ constraint}

Now we discuss the possible constraint on the PBH abundance from the CMB SZ power spectrum measurement. When we fix the PBH mass $M$ and the emission efficiency $\epsilon$, the amplitude of the SZ spectrum is determined by the $\mathrm{PBH}$ abundance $f_{\mathrm{PBH}}$ in our model.

Since the SZ effect due to PBHs can induce the CMB temperature anisotropy on small scales, the small-scale CMB measurement provides the constraint on the PBH abundance. The SPT data reach the minimum value,

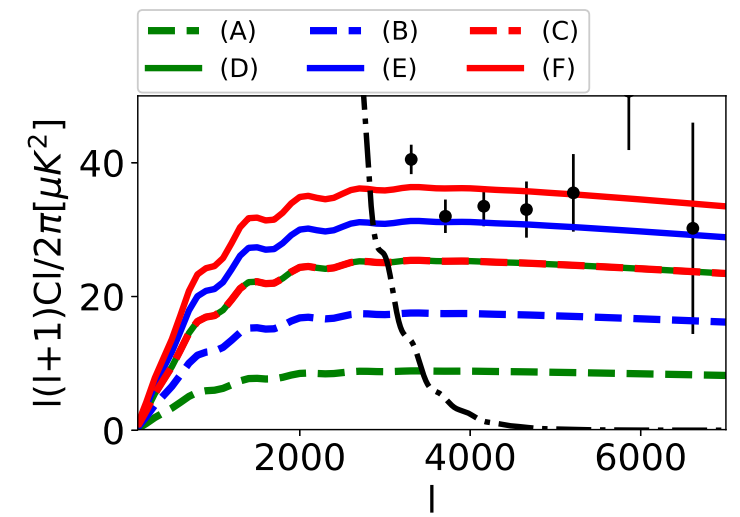

FIG. 4. The angular power spectrum of the CMB temperature caused by the SZ effects from PBHs. The parameter sets for cases $(\mathrm{A})(\mathrm{F})$ are as shown in Table I. The black dasheddotted line is the primordial power spectrum of the CMB temperature and the black circles with error bars represent the SPT data.

$C_{l}^{\mathrm{SPT}}$ at $l_{\mathrm{SPT}}=3709$. Therefore, the condition, $C_{l}^{T T}<$ $C_{l}^{\mathrm{SPT}}$ at $l=l_{\mathrm{SPT}}$ gives the limit on $f_{\mathrm{PBH}}$ with fixing $\epsilon$ and $M$, because of the flat shape of the spectrum. We found that the constraint from the SZ angular power spectrum is roughly independent on the $\mathrm{PBH}$ mass and given in

$$
f_{\mathrm{PBH}}<10^{-3}\left(\frac{\epsilon}{10^{-2}}\right)^{-1},
$$

for $10^{-2} M_{\odot}<M<10^{3} M_{\odot}$. Therefore, when we adopt $\epsilon, 10^{-13}<\epsilon<10^{-7}$ for $1 M_{\odot}<M<10^{3} M_{\odot}$, as in Refs. [28, 29], the current SPT CMB measurement on small scales Eq. (21) cannot provide the constraint on the $\mathrm{PBH}$ abundance. When the $\mathrm{PBH}$ has $\epsilon$ larger than $10^{-5}$ as discussed in Refs. [30, 39], we can obtain the abundance constraint from the SPT data. However, according to Refs. $[28,29]$, we found that the CMB measurement of the global Thomson optical depth provides the $\mathrm{PBH}$ abundance constraint $f_{\mathrm{PBH}}<10^{-9}\left(\epsilon / 10^{-2}\right)^{-1}$ in terms of $\epsilon$. Therefore, we conclude that the constraint from the SZ measurement is always weaker than the one from the global Thomson optical depth.

\section{CONCLUSION}

In this paper, we have investigated the CMB temperature angular power spectrum due to the SZ effect caused by PBHs. The gas accreting on a PBH heats up because of the release of the gravitational energy. As a result, the heated gas in the vicinity of the $\mathrm{PBH}$ emits the UV and $\mathrm{x}$-ray photons. These photons can ionize and heat the IGM around the PBH. The ionized hot IGM causes the secondary CMB temperature anisotropy through the SZ effect. 
First assuming the luminosity of photons emitted in the vicinity of a $\mathrm{PBH}$ with a free parameter, we have evaluated the profiles of the IGM ionization fraction and temperature around a $\mathrm{PBH}$ by solving the one-dimensional radiative transfer equations. Based on these profiles, we have obtained the SZ Compton $y$-parameter profile around a $\mathrm{PBH}$. Following the halo formalism, finally, we have calculated the CMB temperature angular power spectrum due to the $\mathrm{PBH}$ SZ effect with assuming the $\mathrm{PBH}$ abundance.

We have shown that the SZ spectrum due to $\mathrm{PBH}$ could dominate the primordial temperature spectrum on smaller scales than the Silk scale. Since the correlation on such scales is made by $y$ distortions between two different PBHs, the shape of the angular power spectrum depends on the matter power spectrum. We have also found that the amplitude of the spectrum is sensitive to the $\mathrm{PBH}$ abundance and the emission efficiency of the gas accreting on PBHs. In the case of a scale-invariant primordial spectrum, the angular power spectrum $l^{2} C_{l}$ has a flat shape on small scales $l^{2} C_{l} \sim$ $0.5 \times\left(f_{\mathrm{PBH}} / 10^{-2}\right)^{2}\left(\epsilon / 10^{-4}\right)^{2}$. Using $\epsilon$ in Ref. [29], we cannot get the observable signal in current observations such as the SPT. However, $\epsilon$ has a theoretical uncertainty and Refs. $[30,39]$ suggest that PBHs could have $\epsilon$ larger than $10^{-5}$. In this case, PBHs could make the observable signature.

We also investigated the impact for the constraint of the $\mathrm{PBH}$ abundance from the SZ effect. Our obtained constraint on the $\mathrm{PBH}$ fraction to dark matter is $f_{\mathrm{PBH}} \lesssim 10^{-3}\left(\epsilon / 10^{-2}\right)$ for the $\mathrm{PBH}$ mass range $10^{-2} M_{\odot}<\widetilde{M}<10^{3} M_{\odot}$. However, we should note that the CMB constraint from the optical depth provides a much tighter constraint with the same $\epsilon$. Therefore we conclude that the SZ measurement does not give a new constraint on the PBH abundance.

Although the constraint from the SZ angular power spectrum is weaker than that from the optical depth, it is worth mentioning the impact of the future small-scale SZ measurements on the probe of the existence of PBHs. We have shown that the CMB temperature spectrum of the $\mathrm{SZ}$ effect due to $\mathrm{PBHs}$ has the flat spectrum on smaller scales than $l \sim 2000$. This flat spectrum extends up to the scale of the ionized region by the $\mathrm{PBH}$ emission, e.g., roughly $1 \mathrm{kpc}$ in the physical scale for $M=10 M_{\odot}$ with $\epsilon=10^{-4}$. The SZ effect in galaxy clusters can produce large CMB anisotropies on small scales. However, its spectrum has a peak around $l \sim 4000$, and the amplitude decays on higher $l$. Therefore, the detection or nondetection of the flat SZ spectrum on higher $l$ than $l=4000$ gives useful information about the existence of PBHs.

In this work, we have assumed the luminosity from the hot gas in the vicinity of a $\mathrm{PBH}$ with introducing a free parameter for the emission efficiency. References. [28-30] have studied the emission efficiency with the simple assumptions analytically. However, to obtain the emission efficiency consistently, we need to solve the dynamics of gas accreting on a $\mathrm{PBH}$ with considering the backreaction of the luminosity from hot gas. In the next study, we address this issue by numerical simulations and investigate the cosmological effects of the emission from gas accreting on a $\mathrm{PBH}$ in more detail.

\section{ACKNOWLEDGMENTS}

We are grateful to Kenji Hasegawa for his useful comments. This work is supported by JSPS KAKENHI Grants No. 15 K17646 (H.T.) and No. 17 H01110 (H.T.).
[1] G. Steigman and M. S. Turner. Cosmological constraints on the properties of weakly interacting massive particles. Nuclear Physics B, 253:375-386, 1985.

[2] R. D. Peccei and H. R. Quinn. Constraints imposed by CP conservation in the presence of pseudoparticles. Phys. Rev. D, 16:1791-1797, September 1977.

[3] R. D. Peccei and H. R. Quinn. CP conservation in the presence of pseudoparticles. Physical Review Letters, 38:1440-1443, June 1977.

[4] Steven Weinberg. A new light boson? Phys. Rev. Lett., 40:223-226, Jan 1978.

[5] F. Wilczek. Problem of strong $p$ and $t$ invariance in the presence of instantons. Phys. Rev. Lett., 40:279-282, Jan 1978.

[6] Y. Chikashige, R.N. Mohapatra, and R.D. Peccei. Are there real goldstone bosons associated with broken lepton number? Physics Letters B, 98(4):265 - 268, 1981.

[7] B. Carr, F. Kühnel, and M. Sandstad. Primordial black holes as dark matter. Phys. Rev. D, 94(8):083504, October 2016.

[8] Y. B. Zel'dovich and I. D. Novikov. The Hypothesis of
Cores Retarded during Expansion and the Hot Cosmological Model. Soviet Ast., 10:602, February 1967.

[9] S. Hawking. Gravitationally collapsed objects of very low mass. MNRAS, 152:75, 1971.

[10] B. P. Abbott, R. Abbott, T. D. Abbott, and et al. Properties of the Binary Black Hole Merger GW150914. Physical Review Letters, 116(24):241102, June 2016.

[11] B. P. Abbott, R. Abbott, T. D. Abbott, and et al. Gw170104: Observation of a 50-solar-mass binary black hole coalescence at redshift 0.2. Phys. Rev. Lett., 118:221101, Jun 2017.

[12] B. P. Abbott, R. Abbott, T. D. Abbott, and et al. Gw170814: A three-detector observation of gravitational waves from a binary black hole coalescence. Phys. Rev. Lett., 119:141101, Oct 2017.

[13] K. Belczynski, D. E. Holz, T. Bulik, and R. O'Shaughnessy. The first gravitational-wave source from the isolated evolution of two stars in the 40-100 solar mass range. Nature (London), 534:512-515, June 2016.

[14] S. Bird, I. Cholis, J. B. Muñoz, Y. Ali-Haïmoud, 
M. Kamionkowski, E. D. Kovetz, A. Raccanelli, and A. G. Riess. Did LIGO Detect Dark Matter? Physical Review Letters, 116(20):201301, May 2016.

[15] M. Sasaki, T. Suyama, T. Tanaka, and S. Yokoyama. Primordial Black Hole Scenario for the GravitationalWave Event GW150914. Physical Review Letters, 117(6):061101, August 2016.

[16] S. Clesse and J. García-Bellido. The clustering of massive Primordial Black Holes as Dark Matter: Measuring their mass distribution with advanced LIGO. Physics of the Dark Universe, 15:142-147, March 2017.

[17] S. W. Hawking. Particle creation by black holes. Communications in Mathematical Physics, 43:199-220, August 1975.

[18] B. J. Carr, K. Kohri, Y. Sendouda, and J. Yokoyama. New cosmological constraints on primordial black holes. Phys. Rev. D, 81(10):104019, May 2010.

[19] H. Tashiro and N. Sugiyama. Constraints on primordial black holes by distortions of the cosmic microwave background. Phys. Rev. D, 78(2):023004, July 2008.

[20] S. J. Clark, B. Dutta, Y. Gao, L. E. Strigari, and S. Watson. Planck constraint on relic primordial black holes. Phys. Rev. D, 95(8):083006, April 2017.

[21] S. J. Clark, B. Dutta, Y. Gao, Y.-Z. Ma, and L. E. Strigari. $21 \mathrm{~cm}$ limits on decaying dark matter and primordial black holes. Phys. Rev. D, 98(4):043006, August 2018.

[22] D. N. Page and S. W. Hawking. Gamma rays from primordial black holes. Astrophys. J., 206:1-7, May 1976.

[23] B. J. Carr. Some cosmological consequences of primordial black-hole evaporations. Astrophys. J., 206:8-25, May 1976.

[24] J. H. MacGibbon and B. J. Carr. Cosmic rays from primordial black holes. Astrophys. J., 371:447-469, April 1991.

[25] P. Tisserand, L. Le Guillou, C. Afonso, J. N. Albert, J. Andersen, R. Ansari, É. Aubourg, P. Bareyre, J. P. Beaulieu, X. Charlot, C. Coutures, R. Ferlet, P. Fouqué, J. F. Glicenstein, B. Goldman, A. Gould, D. Graff, M. Gros, J. Haissinski, C. Hamadache, J. de Kat, T. Lasserre, É. Lesquoy, C. Loup, C. Magneville, J. B. Marquette, É. Maurice, A. Maury, A. Milsztajn, M. Moniez, N. Palanque-Delabrouille, O. Perdereau, Y. R. Rahal, J. Rich, M. Spiro, A. Vidal-Madjar, L. Vigroux, S. Zylberajch, and EROS-2 Collaboration. Limits on the Macho content of the Galactic Halo from the EROS-2 Survey of the Magellanic Clouds. A\&A A, 469:387404, July 2007.

[26] H. Niikura, M. Takada, N. Yasuda, R. H. Lupton, T. Sumi, S. More, T. Kurita, S. Sugiyama, A. More, M. Oguri, and M. Chiba. Microlensing constraints on primordial black holes with the Subaru/HSC Andromeda observation. arXiv e-prints, January 2017.

[27] M. Zumalacárregui and U. Seljak. Limits on StellarMass Compact Objects as Dark Matter from Gravita- tional Lensing of Type Ia Supernovae. Physical Review Letters, 121(14):141101, October 2018.

[28] M. Ricotti, J. Ostriker, and K. Mack. Effect of Primordial Black Holes on the Cosmic Microwave Background and Cosmological Parameter Estimates. page U5.004, January 2017.

[29] Y. Ali-Haïmoud and M. Kamionkowski. Cosmic microwave background limits on accreting primordial black holes. Phys. Rev. D, 95(4):043534, February 2017.

[30] Vivian Poulin, Pasquale D. Serpico, Francesca Calore, Sebastien Clesse, and Kazunori Kohri. CMB bounds on disk-accreting massive primordial black holes. Phys. Rev., D96(8):083524, 2017.

[31] J. L. Bernal, N. Bellomo, A. Raccanelli, and L. Verde. Cosmological implications of primordial black holes. J. Cosmology Astropart. Phys., 10:052, October 2017.

[32] H. Tashiro and N. Sugiyama. The effect of primordial black holes on 21-cm fluctuations. MNRAS, 435:30013008, November 2013.

[33] J.-O. Gong and N. Kitajima. Small-scale structure and $21 \mathrm{~cm}$ fluctuations by primordial black holes. J. Cosmology Astropart. Phys., 8:017, August 2017.

[34] Y. B. Zeldovich and R. A. Sunyaev. The Interaction of Matter and Radiation in a Hot-Model Universe. Ap $\& S S$, 4:301-316, July 1969.

[35] R. A. Sunyaev and Y. B. Zeldovich. The Observations of Relic Radiation as a Test of the Nature of X-Ray Radiation from the Clusters of Galaxies. Comments on Astrophysics and Space Physics, 4:173, November 1972.

[36] D. J. Fixsen, E. S. Cheng, J. M. Gales, J. C. Mather, R. A. ShaFer, and E. L. Wright. The cosmic microwave background spectrum from the full cobe firas data set. The Astrophysical Journal, 473(2):576, 1996.

[37] R. Chown, Y. Omori, K. Aylor, B. A. Benson, L. E. Bleem, and et al. Maps of the Southern Millimeter-wave Sky from Combined $2500 \mathrm{deg}^{2}$ SPT-SZ and Planck Temperature Data. ApJS, 239:10, November 2018.

[38] Planck Collaboration and N. et al. Aghanim. Planck 2018 results. VI. Cosmological parameters. ArXiv e-prints, July 2018.

[39] José Luis Bernal, Alvise Raccanelli, Licia Verde, and Joseph Silk. Signatures of primordial black holes as seeds of supermassive black holes. Journal of Cosmology and Astro-Particle Physics, 2018:017, May 2018.

[40] D. Giannios, N. D. Kylafis, and D. Psaltis. Spectra and time variability of Galactic black-hole X-ray sources in the low/hard state. A\&A, 425:163-169, October 2004.

[41] M. Fukugita and M. Kawasaki. Reionization during Hierarchical Clustering in a Universe Dominated by Cold Dark Matter. MNRAS, 269:563, August 1994.

[42] E. Komatsu and T. Kitayama. Sunyaev-Zeldovich Fluctuations from Spatial Correlations between Clusters of Galaxies. ApJ, 526:L1-L4, November 1999.

[43] Uroš Seljak. Analytic model for galaxy and dark matter clustering. MNRAS, 318:203-213, October 2000. 